\title{
FEBRE NA INFÂNCIA: CONHECIMENTO, PERCEPÇÃO E ATITUDE MATERNA
}

\author{
FEVER IN CHILDHOOD: MOTHERS' KNOWLEDGE, PERCEPTION AND ATTITUDE
}

\author{
Cristiane Tomaz Rocha \\ Rômulo Rocha Regis " \\ Paulo Nelson-Filho \\ Alexandra Mussolino de Queiroz
}

\section{RESUMO}

Introdução: $\mathrm{O}$ objetivo deste trabalho é determinar o conhecimento, a percepção e a atitude de mães diante da febre de suas crianças, atendidas na Disciplina de Odontopediatria da Faculdade de Odontologia de Ribeirão Preto-USP. Métodos: Sessenta mulheres, com faixa etária entre 17 e 45 anos foram selecionadas. Um questionário foi aplicado, incluindo-se perguntas sobre as causas, consequências e cuidados domiciliares com a febre. Informaçóes adicionais sobre o uso de medicamentos para tratamento da febre e efeitos colaterais decorrentes também foram obtidas. Os dados foram agrupados e submetidos à análise percentual. Resultados: Para detectar a presença de febre, $58(96,7 \%)$ mães tocavam a pele da criança em locais como testa $(58,3 \%)$ e pescoço $(31,7 \%)$ e $46(76,7 \%)$ das mães utilizavam o termômetro. Todas as máes relataram que febre alta pode provocar problemas graves de saúde, como convulsão (90\%), delírio (66,7\%) e perda de peso $(55 \%)$. Antipirético era sempre oferecido às crianças com febre, mas antibióticos náo eram administrados $(96,7 \%) .71,6 \%$ das mães acreditavam que antibióticos provocam efeitos colaterais, principalmente alergia $(46,7 \%)$ e alteraçôes nos dentes (86,7\%), como a cárie dentária (63,3\%). Conclusão: Assim, apesar da crescente ênfase em educação em saúde, informaçôes básicas sobre febre são necessárias para reduzir o medo e ansiedade das mães.

DESCRITORES: Febre • Criança • Conhecimento * Percepção • Atitude

\section{ABSTRACT}

Introduction: The aim of this study is to determine mothers' knowledge, perception and attitude against the fever of their children, attended the Discipline of Pediatric Dentistry, School of Dentistry of Ribeirão Preto-USP. Methods: Sixty women, aged between 17 and 45 years were selected. A questionnaire was administered, including questions about the causes, consequences and care home with fever. Additional information about the use of medicines to treat fever and caused side effects also were obtained. Data were grouped and submitted to analysis percentage. Results: To detect the presence of fever, 58 (96.7\%) mothers played the skin of children in places like forehead (58.3\%) and neck (31.7\%) and $46(76.7 \%)$ mothers using the thermometer. All mothers reported that high fever can cause serious health problems such as seizures (90\%), delirium (66.7\%) and weight loss (55\%). Antipyretic was always offered to children with fever, but antibiotics were not administered (96.7\%). 71.6\% mothers believed that antibiotics cause side effects, especially allergic (46.7\%) and changes in teeth (86.7\%), such as dental caries (63.3\%). Conclusion: Thus, despite the increasing emphasis on health education, basic information about fever are necessary to reduce mothers' fear and anxiety.

DESCRIPTORS: Fever $\bullet$ Child $\bullet$ Knowledge $\bullet$ Perception $\bullet$ Attitude

\footnotetext{
* Mestre e Doutoranda em Odontopediatria da Faculdade de Odontologia de Ribeirão Preto-USP.E-mail:cristianerocha@forp.usp.br.

** Mestre e Doutorando em Reabilitação Oral da Faculdade de Odontologia de Ribeirão Preto-USP. E-mail: romuloregis@hotmail.com.

*** Professor Titular da Disciplina de Odontopediatria da Faculdade de Odontologia de Ribeiráo Preto-USP. E-mail: nelson@forp.usp.br.

**** Professora Doutora da Disciplina de Odontopediatria da Faculdade de Odontologia de Ribeirão Preto-USP. E-mail: amqueiroz@forp.usp.br.
} 


\section{INTRODUÇÃO}

A temperatura orgânica é controlada por um centro termo-regulatório no hipotálamo, através de um sistema de "feed-back" (Dinarelloe wolff'12 1978). Uma alteração desse controle ocasiona a febre, um dos sinais mais comuns de doenças em crianças (Adam e Stankov ${ }^{1}$ 1994), que ocorre na presença de pirógenos (Aronoff e Neilson ${ }^{5}$ 2001), sendo considerada como uma resposta normal do organismo no combate às infecçóes (Kluger ${ }^{17}, 1992$; Sarrell et al. $.^{24}, 2002$; Roth et al. $\left.{ }^{23}, 2004\right)$. Essa ação se dá pelo retardo do crescimento e da reprodução de bactérias e vírus, aumento da produçáo de neutrófilos e proliferação de células- $T$, ajudando na reação de fase aguda do organismo (Adam², 1996).

A febre constitui uma das maiores preocupaçóes para os pais quando seus filhos encontram-se doentes ( $\mathrm{Kai}^{15}$, 1996), gerando uma grande procura por cuidados médicos, devido à extrema ansiedade com relaçáo aos possíveis problemas que ela possa ocasionar (Kramer et al. ${ }^{18}$, 1985). Schmitt ${ }^{25}$ (1980) descreveu, pela primeira vez, a fobia da febre, ou seja, o medo excessivo dos pais diante da febre de suas crianças, independente do grau de instrução e classe econômica (Kramer et al. $\left.{ }^{18}, 1985\right)$. Estudos atuais ainda relatam a permanência dessa fobia, levando a preocupação e tratamentos desnecessários (Crocetti et al. ${ }^{11}$, 2001; Al-Nouri e Basheer ${ }^{4}, 2006$ ).

Al-Nouri e Basheer ${ }^{4}$ (2006) avaliaram o conhecimento e a atitude de mães sobre a febre em seus filhos. Os resultados mostraram que a maioria delas acreditava que a febre poderia provocar danos como paralisia cerebral, retardo mental, convulsão, delírio e morte. Dentre as causas da febre, foram citadas infecção, exposição ao frio e/ou ao sol, processo de erupção dentária e ingestão de bebidas quentes. Para reduzir a febre, a maioria administrava antipiréticos, principalmente o paracetamol. Além disso, foi observado o uso indiscriminado de antibióticos.

A falta de informação faz com que medicamentos desnecessários sejam prescritos, com o intuito de reduzir o desconforto da criança e a ansiedade dos pais (Adam e Stankov ${ }^{1}$, 1994). Na ausência de outros sinais e sintomas, os pediatras, por meio de medidas educacionais (Casey et al. ${ }^{9}, 1984 ; \mathrm{Kai}^{15}$, 1996), deveriam tranquilizar os pais (Baraff et al. ${ }^{6}, 1993$ ), recomendando outros meios para reduçáo da temperatura, que podem incluir tratamentos não-farmacológicos.

A baixa compreensão em torno desse assunto revela a necessidade de mais pesquisas que determinem com precisão o que os pais sabem sobre febre, o grau de ansiedade e medo associados e a forma como essa alteração é tratada, para que, a partir desses dados, programas voltados à instrução da comunidade sejam criados.

Assim, o objetivo deste trabalho é determinar o conhecimento, a percepção e a atitude de mães diante da febre de suas crianças, atendidas na disciplina de Odontopediatria da Faculdade de Odontologia de Ribeirão Preto, por meio da aplicação de um questionário.

\section{MÉTODOS}

A amostra foi composta por 60 mães cujos filhos estavam em tratamento odontológico na Faculdade de Odontologia de Ribeirão Preto (FORP), Universidade de São Paulo, São Paulo, Brasil, sob supervisão da disciplina de Odontopediatria, do Departamento de Clínica Infantil e Odontologia Preventiva e Social. Foram incluídas mães de crianças que apresentaram, no mínimo, um episódio de febre.

Após aprovação do projeto pelo Comitê de Ética em Pesquisa da FORP, sob o número de processo 2007.1.424.58.5, as mães assinaram um termo de consentimento livre e esclarecido concordando com a participação na pesquisa.

A seguir, um único pesquisador aplicou um questionário com respeito ao conhecimento, a percepçáo e o manejo das mães diante da febre de suas crianças (Quadro I), onde os voluntários assinalavam uma ou mais alternativas apresentadas para cada pergunta. Ao final da aplicação do questionário, as mães foram reunidas na sala de espera da clínica e receberam informaçóes relativas à febre. Posteriormente, os dados coletados foram submetidos à análise percentual.

\section{RESULTADOS}

Com relação ao número de filhos, a maioria das mães participantes possuíam $1(35 \%)$ ou $2(36,7 \%)$ filhos e a idade delas variou entre 15 e 45 anos, com média de $29,7( \pm 7,5)$. Quanto ao grau de escolaridade, $24(40 \%)$ possuíam $1^{\circ}$ grau incompleto, $14(23,3 \%)$ possuíam $1^{\circ}$ grau completo, $6(10 \%)$ possuíam $2^{\circ}$ grau incompleto e $16(26,7 \%), 2^{\circ}$ grau completo. A temperatura acima da qual as mães consideravam suas crianças com febre foi de $38^{\circ} \mathrm{C}$ para $19(31,7 \%)$ e de $37,5^{\circ} \mathrm{C}$ para $12(20 \%)$ delas. Para detectar a presença de febre, $58(96,7 \%)$ tocavam a pele da criança em locais como testa $(58,3 \%)$ e pescoço $(31,7 \%)$ e $46(76,7 \%)$ utilizavam o termômetro.

Quando questionadas sobre as causas da febre, apro- 
ximadamente $90 \%$ das mães apontaram a infecção (55) e o resfriado (53) como as principais. Com relação aos efeitos provocados pela febre, a redução do apetite(x) e a apatia $(\mathrm{x})$ foram relatadas como os mais frequentemente ocasionados. Todas as participantes (100\%) relataram que a febre alta poderia ocasionar problemas mais graves, como convulsão $(90 \%)$, delírio $(66,7 \%)$ e perda de peso $(55 \%)$.

Dentre as entrevistadas, $35(58,3 \%)$ levavam suas crianças para serem consultadas por um profissional de saúde algumas horas após o surgimento da febre. A maioria das mães $(68,3 \%)$ procurava atendimento médico no posto de saúde.

Banho morno $(76,6 \%)$ e diminuição da quantidade de roupas $(71,6 \%)$ foram citados como medidas para a redução da temperatura. Todas as mães (100\%) administravam antipiréticos aos seus filhos, sendo a dipirona $(73,3 \%)$ e o paracetamol $(45 \%)$ os mais utilizados. Quando questionadas se esses remédios poderiam provocar efeitos prejudiciais à criança, $30(50 \%)$ delas acreditavam que náo. Dentre estes efeitos prejudiciais, as mães acreditavam que vômitos (40\%), alergias $(33,3 \%)$ e náuseas $(40 \%)$, podiam ocorrer.

Apenas 2 mães $(3,3 \%)$ relataram que utilizavam antibióticos para tratar seus filhos febris, sendo a amoxicilina o fármaco escolhido em ambos os casos. Das entrevistadas, $71,6 \%$ achavam que os antibióticos eram capazes de provocar efeitos colaterais, tais como alergia $(46,7 \%)$ e erupçóes na pele $(21,6 \%)$. Além disso, 52 mães $(86,7 \%)$ relataram que antibióticos podem provocar alteraçóes nos dentes, como cárie $(63,3 \%)$ e manchas $(51,6 \%)$.

\section{DISCUSSÃO}

A febre é um problema comum que acomete a criança e que, geralmente, preocupa os pais, cujos conceitos equivocados podem levá-los a tratamentos inapropriados, bem como a utilização exagerada e desnecessária de serviços de saúde (Schmitt ${ }^{25}$, 1980; Casey et al. ${ }^{9}$, 1984; Al-Eissa et al. ${ }^{3}$, 2000; McErlean et al..$^{22}$, 2001; Goldman e Scolnik $\left.{ }^{13}, 2004\right)$. A média de idade das entrevistadas era de 29,7 $( \pm 7,5)$ anos, nenhuma havia cursado faculdade e a maioria delas possuía ensino fundamental incompleto (40\%). De acordo com o estudo de Impicciatore et al.14 (1998), preocupação exagerada de mães diante da febre está associada com um baixo nível educacional, concordando com os achados deste trabalho. Além disso, a maioria das mães possuía 1 (35\%) ou 2 (36,7\%) filhos, o que também sugere pouca experiência sobre febre em suas crianças.

Em um estudo tipo meta-análise, Teng et al.28 (2007) avaliaram a importância da palpação na detecção da febre em crianças. Os resultados revelaram uma sensibilidade de $89,2 \%$ e especificidade de $50 \%$, o que registra que esse método pode ser muito útil para descartar a presença desse sinal. No entanto, no presente estudo, 58 (96,7\%) mães tocavam a pele da criança considerando essa manobra uma forma de detectar a febre, demonstrando a necessidade de informá-las a respeito de métodos mais precisos na identificação da febre. Achados semelhantes foram descritos por Al-Nouri e Basheer ${ }^{4}$ (2006).

A temperatura fisiológica do organismo varia de 35 a $37,2^{\circ} \mathrm{C}$ (Singhi et al. ${ }^{26}, 1991$; Blumenthal $\left.{ }^{7}, 1998\right)$. No presente estudo, 24 (40\%) mães consideravam apenas aferiçóes acima de $38^{\circ} \mathrm{C}$ como presença de febre, mostrando, assim, um conceito incorreto quanto ao diagnóstico da febre.

Em relação à etiologia da febre, a infecção foi apontada por $91,7 \%$ (55) das entrevistadas como a principal causa, concordando com Al-Nouri e Basheer ${ }^{4}$ (2006), onde $96 \%$ das mães reconheceram a infecção como principal fator etiológico da febre. É consenso entre os pediatras que mais de $90 \%$ das febres agudas em crianças são oriundas de infecçóes virais e requerem apenas terapia de suporte (Casey et al. ${ }^{9}, 1984$ ), como repouso em sala bem ventilada, aumento na ingestáo de líquido e compressas com água morna (Crocetti et al. ${ }^{11}, 2001$ ).

Erupção dental foi relatada por 35 mães $(58,3 \%)$ como responsável pelo surgimento da febre. O estudo de Al-Nouri e Basheer ${ }^{4}$ (2006) relataram que 85\% das mães consideraram a erupção como a causa da febre. Entretanto, Macknin et al. ${ }^{20}$, em 2000, não encontraram associação significante entre erupção dental e febre em crianças.

A perda de apetite (91\%) e o choro excessivo (51\%) foram considerados os principais efeitos provocados pela febre, assim como reportado por Al-Nouri e Basheer ${ }^{4}$ (2006). Quando questionadas sobre os possíveis danos graves da febre alta, a convulsão foi o mais citado (90\%), o que concorda com outros estudos (Kramer et al. ${ }^{18}$, 1985; May e Bauchner ${ }^{21}$, 1992; Al-Eissa et al. ${ }^{3}, 2000$; Crocetti et al. ${ }^{11}$, 2001; Al-Nouri e Basheer ${ }^{4}, 2006$ ).

Este estudo revelou que $100 \%$ das crianças recebiam antipiréticos, dentre eles a dipirona $(73,3 \%)$ e o paracetamol (45\%), semelhante aos achados de Impicciatore et al..$^{14}$ (1998) e Linder et al. ${ }^{19}$ (1999), segundo as quais 
$88 \%$ e $97,9 \%$ das crianças, respectivamente, eram tratadas com essas drogas. A redução da temperatura corporal e potenciais complicaçôes não têm sido as principais indicações desses medicamentos pelos pediatras (May e Bauchner ${ }^{21}$, 1992), mas o alívio do desconforto da criança, bem como da ansiedade dos pais (Kinmonth et al. ${ }^{16}$, 1992). De acordo com Connell ${ }^{10}$ (1997), somente temperaturas de $40^{\circ} \mathrm{C}$ ou maiores deveriam ser reduzidas por meio de medicamentos, portanto a administração de antipirético deveria ser individualizada e baseada em outros sinais além da temperatura. Um achado interessante foi que embora a maioria das máes administrasse antipiréticos às crianças febris, a grande maioria delas $(71,6 \%)$ acreditava que esses medicamentos poderiam causar efeitos colaterais como alergia (25\%), vômitos $(23,3 \%)$ e náuseas $(16,6 \%)$. Assim, juntamente com o uso desnecessário de medicamentos, a má compreensão quanto ao manejo da criança febril pode ser reforçada pelo fato de 58,3\% (35) das mães terem relatado que levavam seus filhos ao médico logo após algumas horas do aparecimento da febre.

No presente estudo, 2 mães $(3,3 \%)$ administravam antibiótico a crianças febris sem conhecimento exato da causa e sem prescrição médica, demonstrando o uso indiscriminado desse medicamento e a ignorância materna com relação ao assunto. $\mathrm{O}$ principal efeito colateral dessa droga citado foi alergia $(46,7 \%)$, concordando com AlNouri e Basheer ${ }^{4}$ (2006).

Quando questionadas se os antibióticos causariam alterações nos dentes, 52 mães $(86,7,3 \%)$ responderam positivamente. Dentre as alteraçóes, $63,3 \%$ citaram a cá-

\begin{tabular}{|l|l|}
\hline Questôes & Alternativas \\
\hline \multirow{2}{*}{$\begin{array}{l}\text { Q1. Quantos filhos } \\
\text { você têm? }\end{array}$} & A. Um; \\
& B. Dois; \\
& C. Três; \\
& D. Mais de três. \\
\hline $\begin{array}{l}\text { Q2.Como você de- } \\
\text { tecta que sua criança } \\
\text { está com febre? }\end{array}$ & A. Sentindo a pele da criança; \\
\hline & B. Termômetro; \\
& A. Testros. \\
\hline $\begin{array}{l}\text { Q3. Onde você toca } \\
\text { pra sentir a tempera- } \\
\text { tura da criança? }\end{array}$ & B. Braço; \\
& D. Peito; \\
& E. Barriga; \\
& F. Costas; \\
& G. Outros. \\
\hline
\end{tabular}

rie dental e 51,6\% manchas. Já é descrito na literatura que pais associam o uso frequente de antibacterianos durante a infância ao precário estado de saúde bucal de seus filhos (Silva et al. ${ }^{27}, 2002$ ). Essas associações ocorrem, provavelmente, devido a muitos medicamentos líquidos pediátricos apresentarem açúcares em sua composição, a fim de mascarar o gosto desagradável dos ingredientes ativos (Bradley e Kinirons ${ }^{8}$, 1998). Além disso, a não realização da higiene bucal após a administração de medicamentos agravaria esse efeito.

O questionário foi aplicado em mães cujos filhos não estavam em estado febril. Segundo Al-Eissa et al. ${ }^{3}$ (2000), a opinião colhida dos pais no momento em que seus filhos estão com febre tem maior importância clínica. Assim, um futuro estudo poderia ser realizado no intuito de comparar os resultados das duas situaçóes e verificar se essa possível diferença é significante. Outra limitação deste estudo foi investigar somente o conhecimento, percepção e manejo de mães, não abordando os pais, uma vez que eles também exercem um papel importante no cuidado da saúde das crianças.

\section{CONCLUSÃO}

Este estudo permitiu determinar que as mães possuem pouco conhecimento, percepçáo errônea e atitudes incorretas com relação à febre de seus filhos. Dessa forma, são necessárias mais informações, abordando a importância dessa alteração, manejo, uso de drogas e atitudes comportamentais, no intuito de diminuir as automedicaçôes, consultas médicas desnecessárias e preocupaçôes exageradas.
Q4. A partir de qual temperatura você considera seu filho com febre?

\begin{tabular}{|l|l|}
\hline & A. Tempo frio; \\
& B. Exposição ao sol; \\
Q5. Na sua opinião, & C. Ingestão de bebidas quentes; \\
o que pode causar & D. Nascimento de dente; \\
febre? & E. Infecção; \\
& F. Resfriado; \\
& G. Outros; \\
& H. Não sabe. \\
\hline
\end{tabular}




\begin{tabular}{|c|c|}
\hline $\begin{array}{l}\text { Q6. Quais são os } \\
\text { efeitos que a febre } \\
\text { pode causar? }\end{array}$ & $\begin{array}{l}\text { A. Redução do apetite; } \\
\text { B. Redução da transpiração; } \\
\text { C. Choro excessivo; } \\
\text { D. Rosto ruborizado; } \\
\text { E. Calafrio; } \\
\text { F. Apatia; } \\
\text { G. Intranqüilidade; } \\
\text { H. Outros. } \\
\end{array}$ \\
\hline $\begin{array}{l}\text { Q7. Você acha que a } \\
\text { febre alta pode cau- } \\
\text { sar algum problema } \\
\text { pra saúde? }\end{array}$ & $\begin{array}{l}\text { A. Sim; } \\
\text { B. Não; } \\
\text { C. Não sabe. }\end{array}$ \\
\hline $\begin{array}{l}\text { Q8. Quais proble- } \\
\text { mas a febre alta pode } \\
\text { causar? }\end{array}$ & $\begin{array}{l}\text { A. Convulsão; } \\
\text { B. Cegueira; } \\
\text { C. Desidratação; } \\
\text { D. Delírio; } \\
\text { E. Epilepsia; } \\
\text { F. Lesóes de boca; } \\
\text { G. Paralisia cerebral; } \\
\text { H. Dano cerebral; } \\
\text { I. Perda de peso; } \\
\text { J. Retardo mental; } \\
\text { L. Meningite; } \\
\text { M. Morte; } \\
\text { N. Coma; } \\
\text { O. Outros; } \\
\text { P. Não sabe. }\end{array}$ \\
\hline $\begin{array}{l}\text { Q9. O que você faz } \\
\text { em casa quando a } \\
\text { sua criança está com } \\
\text { febre? }\end{array}$ & $\begin{array}{l}\text { A. Diminui a quantidade de } \\
\text { roupas; } \\
\text { B. Passa água fria ou gelo enrolado } \\
\text { em pano; } \\
\text { C. Aumenta a quantidade de rou- } \\
\text { pas ou cobre com cobertor; } \\
\text { D. Compressa; } \\
\text { E. Banho frio; } \\
\text { F. Remédio; } \\
\text { G. Outros; } \\
\text { H. Não sabe. } \\
\end{array}$ \\
\hline $\begin{array}{l}\text { Q10. Em que mo- } \\
\text { mento você procura } \\
\text { um profissional de } \\
\text { saúde para tratá-la? }\end{array}$ & $\begin{array}{l}\text { A. Após algumas horas; } \\
\text { B. Até um dia; } \\
\text { C. Até } 2 \text { dias; } \\
\text { D. Após } 2 \text { dias; } \\
\text { E. Quando a temperatura está alta; } \\
\text { F. Outros; } \\
\text { G. Não sabe. } \\
\end{array}$ \\
\hline $\begin{array}{l}\text { Q11. Qual tipo de } \\
\text { profissional você } \\
\text { costuma procurar? }\end{array}$ & $\begin{array}{l}\text { A. Farmacêutico; } \\
\text { B. Médico de convênio; } \\
\text { C. Médico particular; } \\
\text { D. Médico de posto; } \\
\text { E. Outros. }\end{array}$ \\
\hline
\end{tabular}

\begin{tabular}{|c|c|}
\hline $\begin{array}{l}\text { Q12. Você dá algu- } \\
\text { ma medicaçáo pra } \\
\text { criança quando está } \\
\text { com febre? }\end{array}$ & $\begin{array}{l}\text { A. Paracetamol; } \\
\text { B. Dipirona; } \\
\text { C. Aspirina; } \\
\text { D. Outros; } \\
\text { E. Não sabe. }\end{array}$ \\
\hline $\begin{array}{l}\text { Q13. Você acredita } \\
\text { que estes remédios } \\
\text { provocam efeitos } \\
\text { prejudiciais? }\end{array}$ & $\begin{array}{l}\text { A. Sim; } \\
\text { B. Não; } \\
\text { C. Não sabe. }\end{array}$ \\
\hline $\begin{array}{l}\text { Q14. Quais efeitos } \\
\text { podem aparecer? }\end{array}$ & $\begin{array}{l}\text { A. Náuseas; } \\
\text { B. Vômito; } \\
\text { C. Alergia; } \\
\text { D. Outros; } \\
\text { E. Não sabe. }\end{array}$ \\
\hline $\begin{array}{l}\text { Q15. Você dá antibi- } \\
\text { ótico à criança? }\end{array}$ & $\begin{array}{l}\text { A. Sim; } \\
\text { B. Não; } \\
\text { C. Não sabe. }\end{array}$ \\
\hline $\begin{array}{l}\text { Q16. Qual antibi- } \\
\text { ótico você passa à } \\
\text { criança? }\end{array}$ & $\begin{array}{l}\text { A. Penicilina Oral; } \\
\text { B. Eritromicina; } \\
\text { C. Amoxicilina; } \\
\text { D. Penicilina injetável; } \\
\text { E. Cefalexina; } \\
\text { F. Azitromicina; } \\
\text { G. Outros; } \\
\text { H. Não sabe. } \\
\end{array}$ \\
\hline $\begin{array}{l}\text { Q17. Você acha que } \\
\text { antibióticos causam } \\
\text { efeitos prejudiciais? }\end{array}$ & $\begin{array}{l}\text { A. Sim; } \\
\text { B. Não; } \\
\text { C. Não sabe. } \\
\end{array}$ \\
\hline $\begin{array}{l}\text { Q18. Quais desses } \\
\text { efeitos podem apa- } \\
\text { recer? }\end{array}$ & $\begin{array}{l}\text { A. Alergia; } \\
\text { B. Paralisia; } \\
\text { C. Erupçốes na pele; } \\
\text { D. Convulsões; } \\
\text { E. Morte; } \\
\text { F. Outros; } \\
\text { G. Não sabe. } \\
\end{array}$ \\
\hline $\begin{array}{l}\text { Q19. Você acha que } \\
\text { antibióticos podem } \\
\text { causar alteraçóes nos } \\
\text { dentes? }\end{array}$ & $\begin{array}{l}\text { A. Sim; } \\
\text { B. Não; } \\
\text { C. Não sabe. }\end{array}$ \\
\hline $\begin{array}{l}\text { Q20. Quais al tera- } \\
\text { çôes podem aparecer } \\
\text { nos dentes? }\end{array}$ & $\begin{array}{l}\text { A. Cárie; } \\
\text { B. Manchamento; } \\
\text { C. Outros; } \\
\text { D. Não sabe. }\end{array}$ \\
\hline
\end{tabular}




\section{REFERÊNCIAS}

1. Adam D, Stankov G. Treatment of fever in childhood. Eur J Pediatr 1994 Jun; 153(6): 394-402.

2. Adam HM. Fever and host responses. Pediatr Rev 1996 Sep; 17(9): 330-1.

3. Al-Eissa YA, Al-Sanie AM, Al-Alola SA, Al-Shaalan MA, Ghazal SS, Al-Harbi AH et al. Parental perceptions of fever in children. Ann Saudi Med 2000 May- Jul; 20(3-4): 202-5.

4. Al-Nouri L, Basheer K. Mother's perceptions of fever in children. J Trop Pediatr 2006 Apr; 52(2): 113-6.

5. Aronoff DM, Neilson EG. Antipyretics: mechanis$\mathrm{ms}$ of action and clinical use in fever suppression. Am J Med 2001 Sep; 111(4): 304-15.

6. Baraff LJ, Bass JW, Fleisher GR, Klein JO, McCracken GH Jr, Powell KR et al. Practice guideline for the management of infants and children 0 to 36 months of age with fever without source. Agency for Health Care Policy and Research. Ann Emerg Med 1993 Jul; 22(7): 1198-210.

7. Blumenthal I. What parents think of fever. Fam Pract 1998 Dec; 15(6): 513-8.

8. Bradley MB, Kinirons MJ. Choice of sugar-free medicines by a sample of dentists, doctors and pharmacists in Northern Ireland: the views of parents and health professionals. Community Dent Health 1998 Jun; 15(2): 105-8.

9. Casey R, McMahon F, McCormick MC, Pasquariello Jr PS, Zavod W, King Jr FH. Fever Therapy: An Educational Intervention for Parents. Pediatrics 1984 May; 73(5): 600-5.

10. Connell F. The causes and treatment of fever: a literature review. Nurs Stand 1997 Dec; 3-9;12(11):40-3.

11. Crocetti M, Moghbeli N, Serwint J. Fever phobia revisited: have parental misconceptions about fever changed in 20 years? Pediatrics 2001 Jun; 107(6): 1241-1246.

12. Dinarello CA, Wolff SM. Pathogenesis of fever in man. N EnglJ Med 1978 Mar; 298(11): 607-12.
13. Goldman RD, Scolnik D. Underdosing of acetaminophen by parents and emergency department utilization. Pediatr Emerg Care 2004 Feb; 20(2): 89-93.

14. Impicciatore $P$, Nannini $S$, Pandolfini $C$, Bonati $M$. Mothers' knowledge of, attitudes toward, and management of fever in preschool children in italy. Preventive Medicine 1998 Mar-Apr; 27(2): 268-73.

15. Kai J. What worries parents when their preschool children are acutely ill, and why: a qualitative study. BMJ 1996 Oct; 19; 313(7063): 983-6.

16. Kinmonth AL, Fulton Y, Campbell MJ. Management of feverish children at home. BMJ 1992 Nov; 305(6862): 1134-6.

17. Kluger MJ. Is fever really a "side-effect" of biological response modifiers? Lancet 1992 Feb: 22; 339(8791):491.

18. Kramer MS, Naimark L, Leduc DG. Parental fever phobia and its correlates. Pediatrics 1985 Jun; 75(6): 1110-3.

19. Linder N, Sirota L, Snapir A, Eisen I, Davidovitch $\mathrm{N}$, Kaplan $\mathrm{G}$ et al. Parental knowledge of the treatment of fever in children. Isr Med Assoc J. 1999 Nov;1(3):158-60.

20. Macknin ML, Piedmonte M, Jacobs J, Skibinski C. Symptoms associated with infant teething: a prospective study. Pediatrics 2000 Apr; 105(4Pt1); 74752.

21. May A, Bauchner H. Fever phobia: the pediatrician's contribution. Pediatrics 1992 Dec; 90(6): 851-4.

22. McErlean MA, Bartfield JM, Kennedy DA, Gilman EA, Stram RL, Raccio-Robak N. Home antipyretic use in children brought to the emergency department. Pediatr Emerg Care 2001 Aug; 17(4): 249-51.

23. Roth J, Zeisberger E, Vybíral S, Janský L. Endogenous antipyretics: neuropeptides and glucocorticoids. Front Biosci 2004 Jan: 1; 9: 916-26.

24. Sarrell M, Cohen HA, Kahan E. Physicians', nurses', and parents' attitudes to and knowledge about fever in early childhood. Patient Educ Couns 2002 Jan; 46(1): 61-5. 
25. Schmitt BD. Fever phobia: misconceptions of parents about fevers. Am J Dis Child 1980 Feb; 134(2): 176-81.

26. Singhi S, Padmini P, Sood V. Urban parents' understanding of fever in children: its dangers, and treatment practices. Indian Pediatr 1991 May; 28(5): 501-5.
27. Silva VS, Silva AN, Maia LC. Antibacterianos pediátricos e cárie dental em crianças: mitos e realidades. Rev Paul Pediatr 2002 Ago; 20(4): 191-5.

28. Teng C, Ng CJ, Nik-Sherina H, Zailinawati AH, Tong SF. The accuracy of mother's touch to detect fever in children: a systematic review. $J$ Trop Pediatr 2008 Fev; 54(1):70-3. Epub 2007 Nov. 25.

Recebido em: 22/4/2009

Aceito em: 1/10/2009 